\title{
A New Approach to Image Denoising by Patch-Based Algorithm
}

\author{
V. Rajanesh ${ }^{1}$, Sreedhar Kollem ${ }^{2}$ \\ Assistant Professor, Department of ECE, JNTUHCES, Sulthanpur, Telangana, India ${ }^{1}$ \\ Assistant Professor, Department of ECE, SR Engineering College, Warangal, Telangana, India ${ }^{2}$
}

\begin{abstract}
Different types of denoising methods are existed in databases. But every method has its own uniqueness. We propose a new method that is adaptive patch based system for image denoising. The approach depends on a pointwise selection of narrow image patches of precise size in the variable neighborhood of each pixel. Our contribution is to engage in each pixel the weighted sum of data points not outside an adaptive neighborhood, in a sense that it balances the efficiency of estimation and the stochastic error, at each contiguous position. In this paper introducing spatial adaptivity, we prolong the work that can be designed as a development of bilateral filtering to image patches. This method is tested by using AWGN and images are taken from databases with different resolutions. The performance of the denoising system is computed in terms of PSNR by taking different noise levels.
\end{abstract}

Keywords: Image denoising; bilateral filtering; mean-squared-error (MSE) estimator; PSNR; Patch based method.

\section{INTRODUCTION}

In recent years, images captured by present-day cameras are habitually corrupted by noise [3]. With increasing pixel resolution but more or less the same aperture size, noise elimination has turn into more relevant. While advances in optics and hardware try to mollify such abominable effects, software-based denoising approaches are more familiar as they are normally device independent and extensively suitable. In the last decade, many such methods have been recommended, lead to extensive enhancement in denoising performance. In [1] and [2], we studied the dispute from an estimation theory aspect to compute the fundamental limits of denoising. The insights gained from that study are applied to promote a hypothetically sound denoising method in this paper. Most of the more competent regularization methods depend on energy functional minimization therefore they are designed to deliberately account for the image geometry, involving the compromise of global weights that weigh the contribution of prior gracefulness terms and a fidelity term [13]. Thus, associated partial differential equations (PDEs) and variationally methods have demonstrated intense results to take up the dispute of edge-preserving smoothing [9], [10], [11], [23], [28]. For reasons of performance and robustness in image processing, other smoothing algorithms cumulative information over a neighborhood of fixed size, based on two primitive criteria: a spatial criterion in order that filtering must be local and a brightness criterion to counterbalance choose only points that are identical in some sense. In view about generic approach, a typical filter is the sigma filter [32]. The well-known nonlinear Gaussian filter [25] defined as

$$
\hat{u}\left(x_{i}\right)=\frac{\sum_{x_{j} \in \Delta_{i}} L_{g}\left(Y_{i}-Y_{j}\right) Y_{j}}{\sum_{x_{j} \in \Delta_{i}} L_{g}\left(Y_{i}-Y_{j}\right)}
$$

Where $\Delta_{i}$ is the window centered at pixel $x_{i}, Y_{i}$ is the observation at $x_{i}$,

$$
L_{g}(z)=\exp \left(\frac{-z^{2}}{2 g^{2}}\right)
$$

and $g$ is a smoothing parameter to be determined and depends on the signal-to-noise ratio (SNR). Finally, if we substitute a Gaussian window to the hard-curved window almost the current position, we get variants of the bilateral filtering.

$$
\hat{u}\left(x_{i}\right)=\frac{\sum_{x_{j} \in \Delta_{i}} L_{g}\left(Y_{i}-Y_{j}\right) K_{h}\left(x_{i}-x_{j}\right) Y_{j}}{\sum_{x_{j} \in \Delta_{i}} L_{g}\left(Y_{i}-Y_{j}\right) K_{h}\left(x_{i}-x_{j}\right)}
$$

where $K_{h}\left(x_{i}-x_{j}\right)$ and $L_{g}\left(Y_{i}-Y_{j}\right)$ are rescaled versions of nonnegative kernel functions $K$ and $L$. In specific, Mrazek brought out the multiple of structural similarities between the iterated bilateral filter.

$\hat{u}_{n+1}\left(x_{i}\right)=\frac{\sum_{x_{j} \in \Delta_{i}} L_{g}\left(\hat{u}_{n}\left(x_{i}\right)-\hat{u}_{n}\left(x_{j}\right)\right) K_{h}\left(x_{i}-x_{j}\right) \hat{u}_{n}\left(x_{j}\right)}{\sum_{x_{j} \in \Delta_{i}} L_{g}\left(\hat{u}_{n}\left(x_{i}\right)-\hat{u}_{n}\left(x_{j}\right)\right) K_{h}\left(x_{i}-x_{j}\right)}$

and local M-smoother

$\hat{u}_{n+1}\left(x_{i}\right)=\frac{\sum_{x_{j} \in \Delta_{i}} w_{g}\left(Y_{j}-\hat{u}_{n}\left(x_{i}\right)\right) K_{h}\left(x_{i}-x_{j}\right) Y_{j}}{\sum_{x_{j} \in \Delta_{i}} w_{g}\left(Y_{j}-\hat{u}_{n}\left(x_{i}\right)\right) K_{h}\left(x_{i}-x_{j}\right)}$ 
Vol. 5, Issue 12, December 2016

where $\quad w_{g}\left(Y_{j}-\hat{u}_{n}\left(x_{i}\right)\right)=\rho_{z}^{1}\left(Y_{j}-\hat{u}_{n}\left(x_{i}\right)\right)$ and $\rho_{z}\left(Y_{j}-\hat{u}_{n}\left(x_{i}\right)\right)$ is the error norm for M-estimators. It is proved that regional $\mathrm{M}$-smoothing uses the initial image in the averaging procedure and determine the minimum of a local criterion considering that iterated bilateral filtering uses the evolving image and must stop subsequently a particular number of iterations in order to avoid a flat image [15]- [20].

To promote better image enhancement algorithms that can supervise structured noise, we need precise models for the many regularities and geometries seen in local patterns. In comparison, a distinct line of work consists then in modelling non-local pairwise interactions from training data [29] or a library of natural image patches [21], [27]. The data points with an identical patch to the central patch will have larger weights in the average as recently proposed by Buades [7], [8] who defined the purported non-local means filter as

$$
\hat{u}\left(x_{i}\right)=\frac{\sum_{x_{j} \in \Delta_{i}} L_{g}\left(Y_{i}-Y_{j}\right) K_{h}\left(x_{i}-x_{j}\right) Y_{j}}{\sum_{x_{j} \in \Delta_{i}} L_{g}\left(Y_{i}-Y_{j}\right) K_{h}\left(x_{i}-x_{j}\right)}
$$

Where $Y_{i}$ denotes a vector of pixel values taken in the neighborhood of a point $x_{i}$. In (5), the similarity between two points $x_{i}$ and $x_{j}$ is based on the Euclidean distance $\left\|Y_{i}-Y_{j}\right\|^{2}$ between two vectorized image patches (see also [58]). It stands observing that, if the size of the patch is reduced to one pixel, the non-local means filter, also controlled by a small number of smoothing parameters and, is closely equivalent to (2). As in [30], [31], [36], we also use narrow image patches (e.g., 7x7 or $9 \times 9$ patches) to enumerate the particular weights therefore they allow capture regional geometric patterns and texels seen in image s. Moreover, we adaptively determine a window (neighborhood) that probably large to balance the accuracy of approximation and the stochastic error, at each spatial position [34]. This transformation method is a moderately change-point detection procedure introduced by Lepskii [33]. The recommended procedure shares some common points with the contemporary non-local means algorithm [17], other patch -based methods [15], [18], [29] and the DUDE algorithm. In [16], the authors design a two-pass approach and substitute the most frequent patch /symbol seen in a local window to the current perverted patch /symbol.

The paper is organized as follows. In Section II, tells about problem statement and introduce the image modeling and some notations. In Section III, we formulate the adaptive weights and patch based algorithm for image denoising. In Section IV, we demonstrate the ability of the method to restore artificially corrupted images with additive white
Gaussian noise (WGN) with different sigma values. Conclusions are presented in Section V.

\section{PROBLEM STATEMENT}

In comparison to exemplar-based approaches for image modeling [14], [19], we propose an unsupervised method that uses no library of image patches and no computational intensive training algorithms [23]. Our adaptive smoothing introduces the joint spatial range domain as the non-local means filter [13] but has a more dominant adaptation to the local structure of the data therefore the size of windows and control parameters are predicted from local image statistics as follows. Consider the following basic image model:

$$
Y_{i}=u\left(x_{i}\right)+\varepsilon_{i}, \quad i=1,2,3 \ldots,|G|
$$

where $x_{i} \in G \subset R^{d}, \quad d \geq 2$ represents the spatial coordinates of the discrete image domain of $|G|$ pixels, and $Y_{i} \in R_{+}$is the observed intensity at location $x_{i}$.

For clarity, an image patch $u_{i}$ is cast as a fixed size square window of $p \times p$ pixels centered at $x_{i}$. In what follows, $u_{i}$ will stand for inadequately a patch or a vector of $p^{2}$ elements where the pixels are concatenated along a fixed lexicographic ordering. As with all patch -based techniques, the size of image patches must be described in advance [31], [32], [35]. Traditionally, the size of the image patch is a parameter-free that specifies how stochastic the user believes the image to be. However, we will see that a patch size of $7 \times 7$ or $9 \times 9$ pixels manage take care of the local geometries and textures in the image while removing unwanted distortions. Finally, the suggested method requires no training step and may be then treated as unsupervised.

\section{IMPLEMENTATION OF ADAPTIVE PATCH BASED SYSTEM}

A. Adaptive Weights

In order to compute the similarity between patches $\hat{u}_{i, n}=\sum_{x_{j} \in \Delta_{i, n}} \pi_{i \sim j, n} Y_{j}$ and $\hat{u}_{j, n}$, a distance must be considered. In [7], [15], [18] several authors showed that the $L_{2}$ distance $\left\|\hat{u}_{i, n}-\hat{u}_{j, n}\right\|^{2}$ is a reliable measure to compare image patches. To make a decision, we have rather used the following normalized distance

$\operatorname{dist}\left(\hat{u}_{i, n-1}, \hat{u}_{j, n-1}\right)=\frac{1}{2}\left[\begin{array}{l}\left(\hat{u}_{i, n-1}-\hat{u}_{j, n-1}\right)^{T} \hat{V}_{i, n-1}^{-1}\left(\hat{u}_{i, n-1}-\hat{u}_{j, n-1}\right)+ \\ \left(\hat{u}_{j, n-1}-\hat{u}_{i, n-1}\right)^{T} \hat{V}_{j, n-1}^{-1}\left(\hat{u}_{j, n-1}-\hat{u}_{i, n-1}\right)\end{array}\right]$

where $\hat{V}_{., n-1}$ is $p^{2} \times p^{2}$ diagonal matrix of the form (the symbol "." is used to denote a spatial position) 


$$
\hat{V}_{., n-1}=\left(\begin{array}{cccc}
\left(\hat{v}_{., n-1}^{(1)}\right)^{2} & 0 & \cdots & 0 \\
0 & \left(\hat{v}_{., n-1}^{(2)}\right)^{2} & \ldots & 0 \\
\vdots & \vdots & \vdots & \vdots \\
0 & \ldots & 0 & \left(\hat{v}_{., n-1}^{\left(p^{2}\right)}\right)^{2}
\end{array}\right)
$$

where $\hat{v}_{,, n-1}^{(l)}, \quad l=1,2, \ldots . . p^{2}$, is the local standard deviation of the estimator $\hat{v}_{., n-1}^{(l)}$, and the index $l$ is used to denote a spatial position in an image patch $\hat{u}_{i, n-1}=\left(\hat{u}_{i, n-1}^{(1)}, \ldots, \hat{u}_{i, n-1}^{(l)}, \ldots, \hat{u}_{i, n-1}^{\left(p^{2}\right)}\right)^{T}$.

Henceforth, we introduce the following commonly used weight function

$$
\pi_{i \sim j, n}=\frac{K\left(\lambda_{\alpha}^{-1} \operatorname{dist}\left(\hat{u}_{i, n-1}, \hat{u}_{j, n-1}\right)\right)}{\sum_{x_{j} \in \Delta_{i, n}} K\left(\lambda_{\alpha}^{-1} \operatorname{dist}\left(\hat{u}_{i, n-1}, \hat{u}_{j, n-1}\right)\right)}
$$

with $K\left(\lambda_{\alpha}^{-1} \operatorname{dist}\left(\hat{u}_{i, n-1}, \hat{u}_{j, n-1}\right)\right)$ denoting a monotone decreasing function, e.g., a kernel $K(z)=\exp (-z / 2)$. Due to the fast decay of the exponential kernel, large distances between predicted patches provoke nearly zero weights. Note that the use of weights enables to relieve the structural hypothesis that the neighborhood is practically modeled by a square window.

B. Patch based image denoising algorithm

i. Let $\left\{p, \alpha, \tilde{\mathrm{n}}, N_{\Delta}\right\}$ be the parameters

ii. Initialization: Compute $\hat{\sigma}^{2}$ and $\left\{\hat{u}_{i, 0}, \hat{u}_{i, 0}^{2}\right\}$ for each $x_{i} \in G$ and set $n=1$

iii. Repeat

- $\quad$ for each $x_{i} \in G$

-Compute

$$
\begin{gathered}
\pi_{i \sim j, n}=\frac{K\left(\lambda_{\alpha}^{-1} \operatorname{dist}\left(\hat{u}_{i, n-1}, \hat{u}_{j, n-1}\right)\right)}{\sum_{x_{j} \in \Delta_{i, n}} K\left(\lambda_{\alpha}^{-1} \operatorname{dist}\left(\hat{u}_{i, n-1}, \hat{u}_{j, n-1}\right)\right)} \\
\hat{u}_{i, n}=\sum_{x_{j} \in \Delta_{i, n}} \pi_{i \sim j, n} Y_{j} \\
\hat{v}_{i, n}^{2}=\hat{\sigma}^{2} \sum_{x_{j} \in \Delta_{i, n}}\left[\pi_{i \sim j, n}\right]^{2}
\end{gathered}
$$

-Choose the window as

$$
\hat{\Delta}\left(x_{i}\right)=\arg \max _{\Delta_{i, n \in N_{\Delta}}}\left\{\begin{array}{l}
\left|\Delta_{i, n}\right|:\left|\hat{u}_{i, n}-\hat{u}_{i, n^{\prime}}\right| \quad \text { for all } \quad 1 \leq n^{\prime}<n \\
\leq \tilde{\mathrm{n}} \hat{u}_{i, n^{\prime}},
\end{array}\right\}
$$

If this rule is violated at iteration $n$, we do not accept $\hat{u}_{i, n}$ and keep the estimate $\hat{u}_{i, n-1}$ as the final estimate $x_{i}$, i.e. $\hat{u}\left(x_{i}\right)=\hat{u}_{i, n-1}$ and $\quad \hat{n}\left(x_{i}\right)=n-1$. This estimate is unchanged at the next iterations and $x_{i}$ is frozen.

- $\quad$ Increment $n$

iv. while $n \leq N_{\Delta}$

\section{EXPERIMENTAL RESULTS}

Our results were measured by the peak signal-to-noise ratio (PSNR) in decibels (dB) defined as $P S N R=10 \log _{10}\left(\frac{255 \times 255}{M S E}\right), M S E=\frac{\sum_{x_{i} \in G}\left(u_{0}\left(x_{i}\right)-\hat{u}\left(x_{i}\right)\right)^{2}}{G}$

where $u_{0}$ is the noise-free original image.

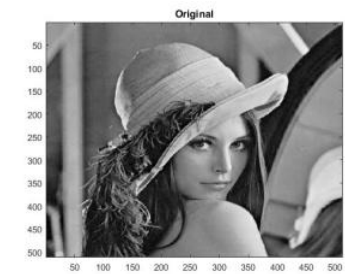

(a) Original Image

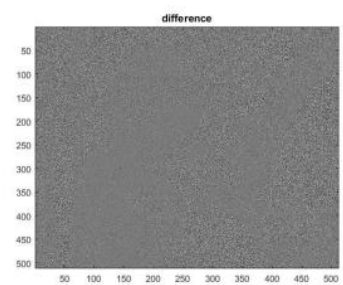

(c) Difference Image

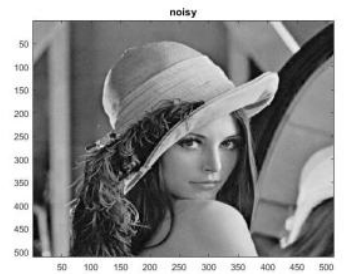

(b) Noisy Image

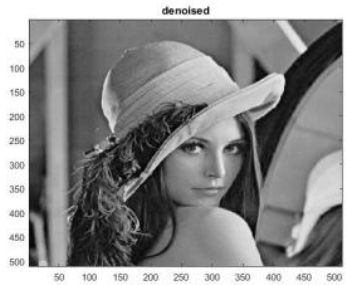

(d) Denoised Image
Fig. 1. Illustration of LENA image at sigma=2

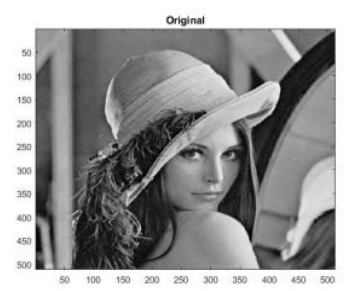

(a) Original Image

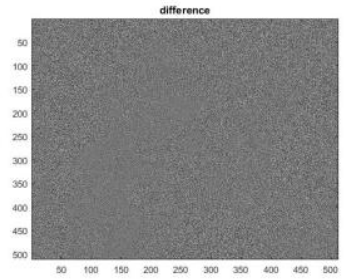

(c) Difference Image

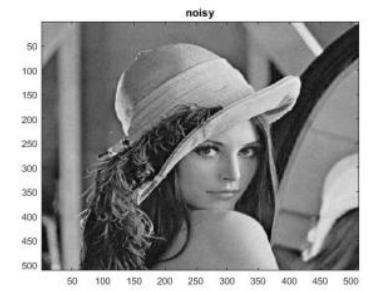

(b) Noisy Image

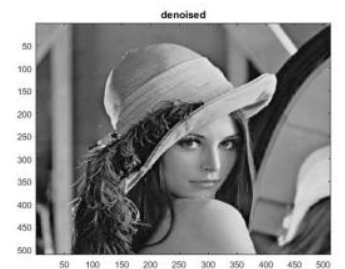

(d) Denoised Image
Fig.2. Illustration of LENA image at sigma=4 
Vol. 5, Issue 12, December 2016

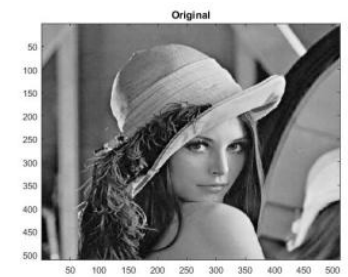

(a) Original Image

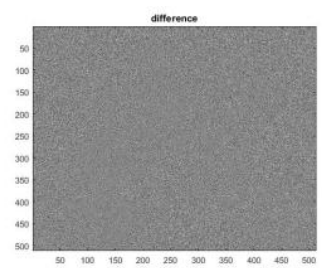

(c) Difference Image

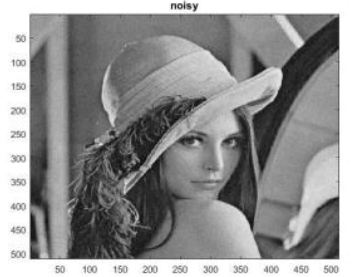

(b) Noisy Image

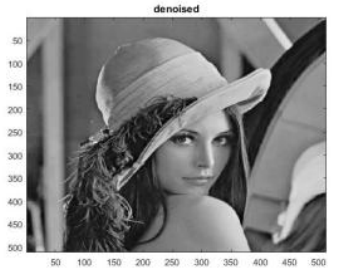

(d) Denoised Image
Fig.3. Illustration of LENA image at sigma=7

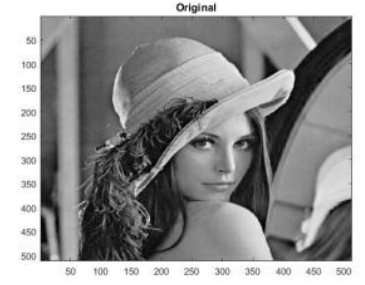

(a) Original Image

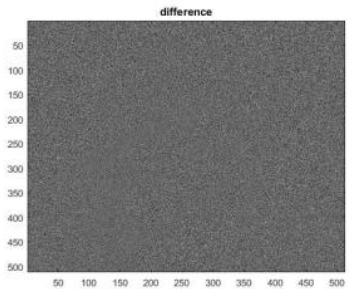

(c) Difference Image

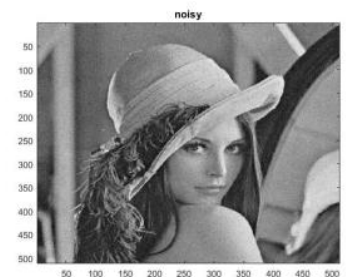

(b) Noisy Image

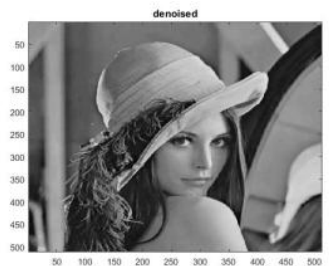

(d) Denoised Image
Fig. 4. Illustration of LENA image at sigma=9

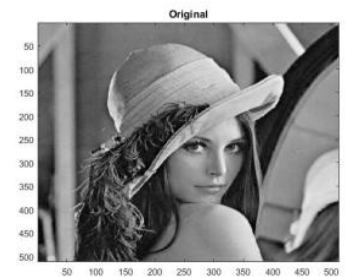

(a) Original Image

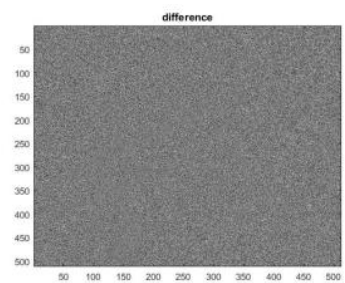

(c) Difference Image

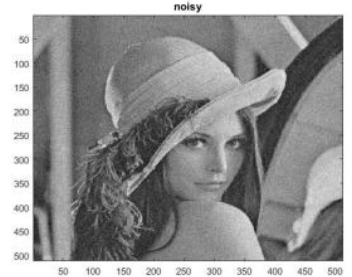

(b) Noisy Image

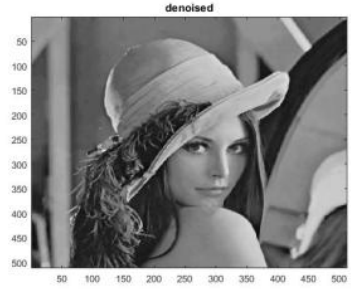

(d) Denoised Image
Fig.5. Illustration of LENA image at sigma $=15$

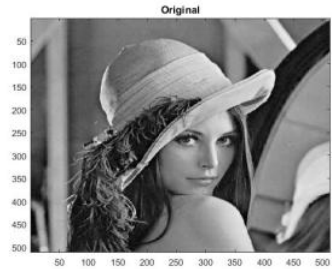

(a) Original Image

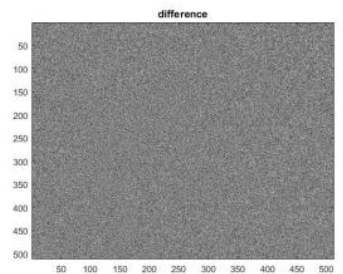

(c) Difference Image

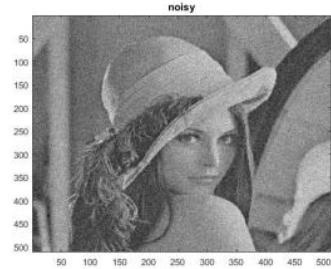

(b) Noisy Image

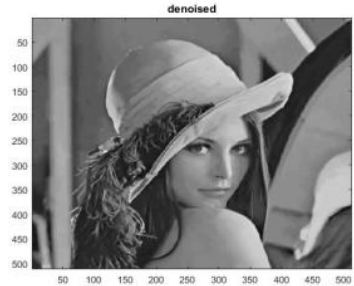

(d) Denoised Image

Fig.6. Illustration of LENA image at sigma=25

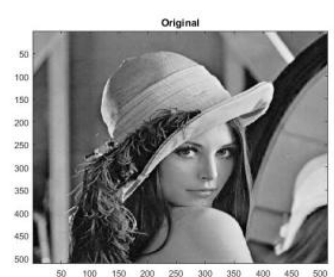

(a) Original Image

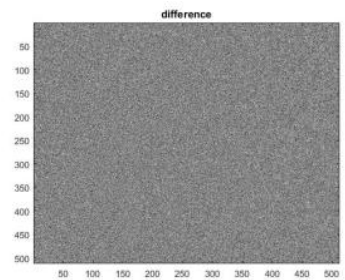

(c) Difference Image

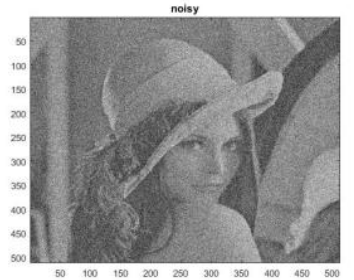

(b) Noisy Image

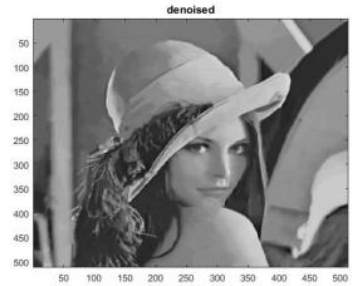

(d) Denoised Image
Fig.7. Illustration of LENA image at sigma=45

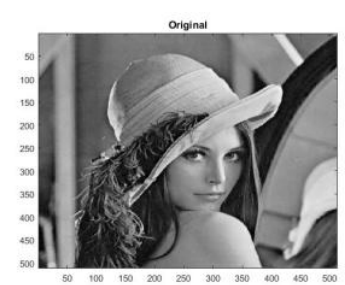

(a) Original Image

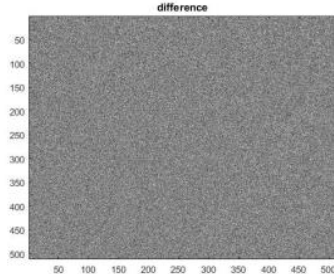

(c) Difference Image

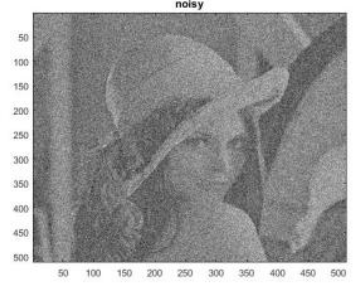

(b) Noisy Image

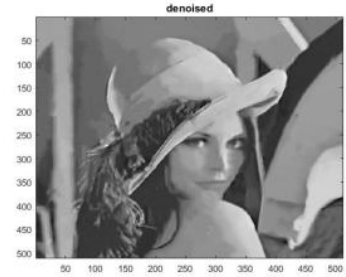

(d) Denoised Image
Fig.8. Illustration of LENA image at sigma=70 
Vol. 5, Issue 12, December 2016

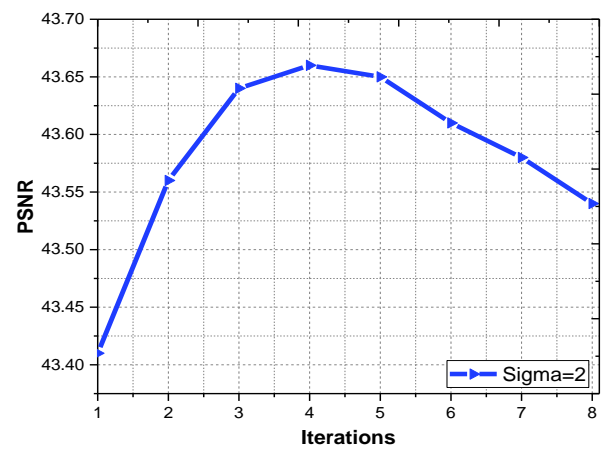

(a) PSNR at sigma $=2$

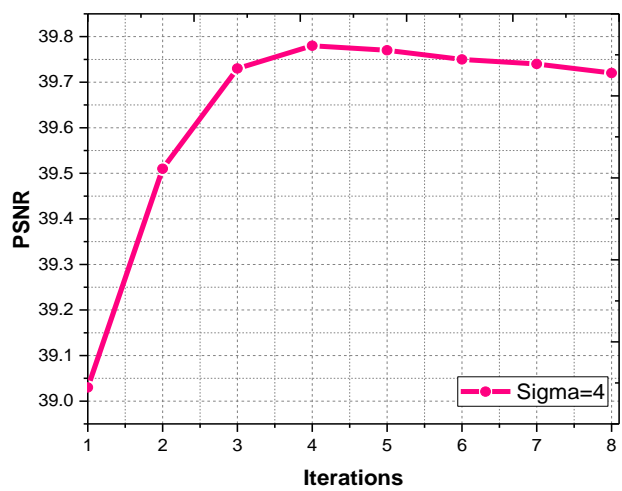

(b) PSNR at sigma $=4$

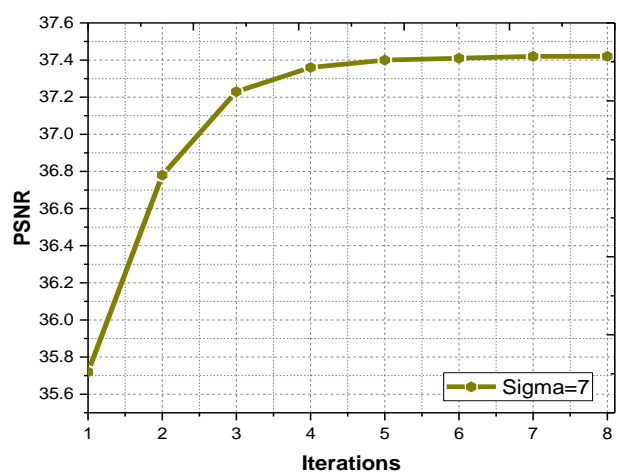

(c) PSNR at sigma=7

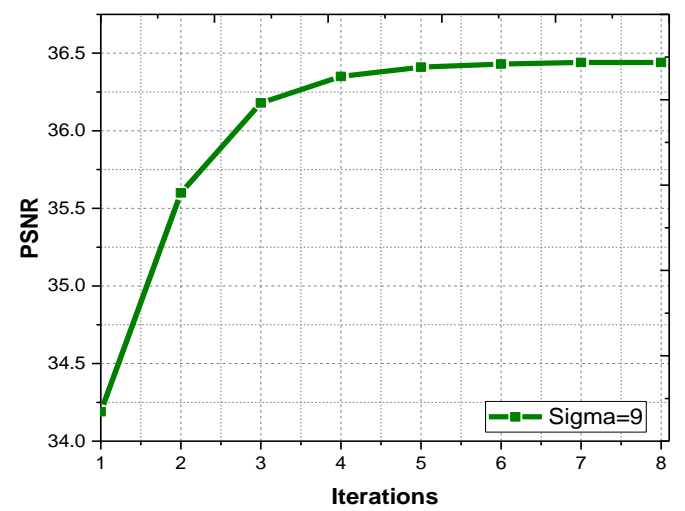

(d) PSNR at sigma $=9$

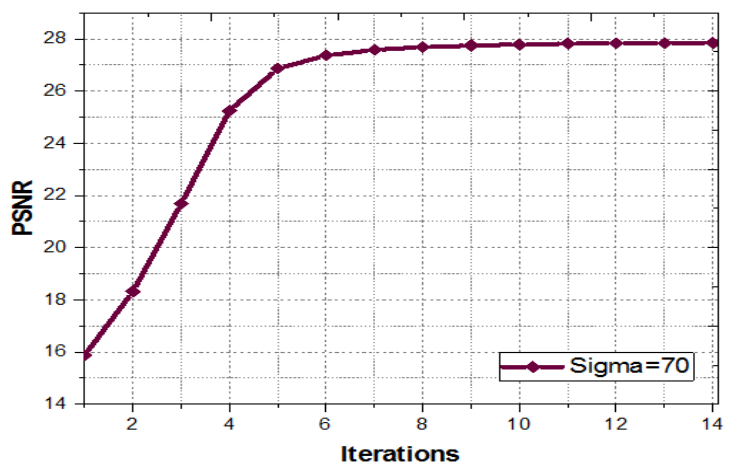

(h) PSNR at sigma $=70$

Fig. 9. Different PSNR values of LENA image at different noise levels

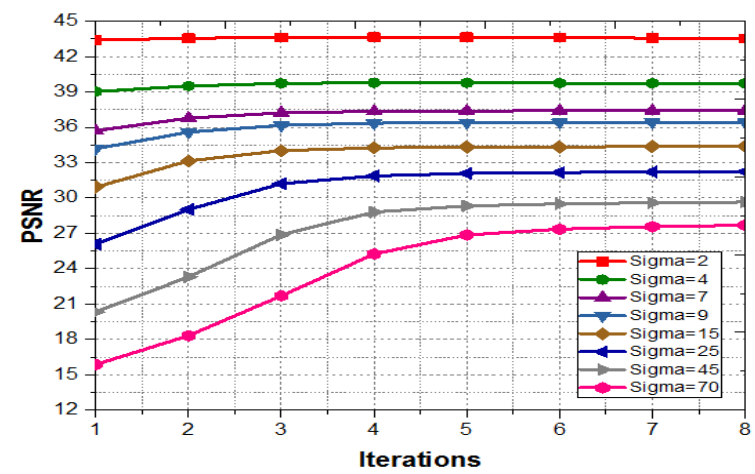

(a) PSNR values of LENA at different sigma value

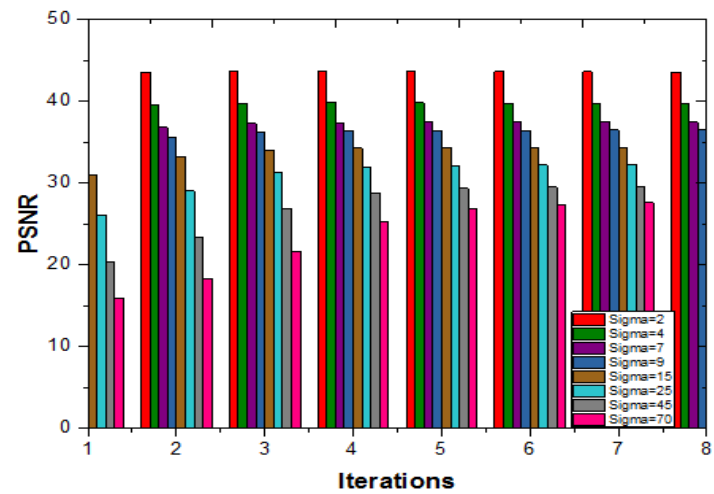

(b) Histogram representation of LENA at different sigma values

Fig10 Comparison of LENA image at different noise levels

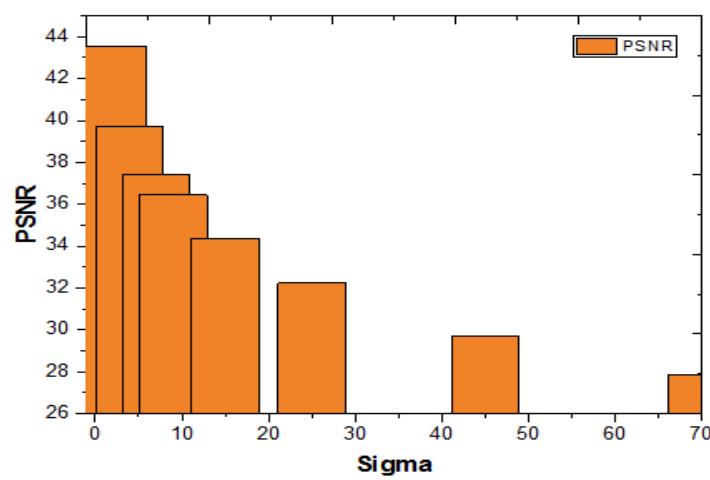

Fig.11. Histogram representation of PSNR versus sigma 
Vol. 5, Issue 12, December 2016

TABLE 1: DENOISING RESULTS OF LENA IMAGE BY TAKING DIFFERENT SIGMA VALUES

\begin{tabular}{|c|c|c|c|c|c|c|c|c|}
\hline $\begin{array}{c}\text { Iterati } \\
\text { ons }\end{array}$ & Sigma=2 & Sigma=4 & Sigma=7 & Sigma=9 & Sigma=15 & Sigma=25 & Sigma=45 & Sigma=70 \\
\hline 1 & 43.41 & APBS & APBS & APBS & APBS & APBS & APBS & APBS \\
\hline 2 & 43.56 & 39.51 & 35.72 & 34.19 & 30.93 & 26.08 & 20.33 & 15.87 \\
\hline 3 & 43.64 & 39.73 & 37.23 & 36.18 & 34.03 & 31.22 & 26.88 & 21.69 \\
\hline 4 & 43.66 & 39.78 & 37.36 & 36.35 & 34.25 & 31.88 & 28.81 & 25.25 \\
\hline 5 & 43.65 & 39.77 & 37.40 & 36.41 & 34.33 & 32.09 & 29.33 & 26.87 \\
\hline 6 & 43.61 & 39.75 & 37.41 & 36.43 & 34.35 & 32.17 & 29.51 & 27.36 \\
\hline 7 & 43.58 & 39.74 & 37.42 & 36.44 & 34.36 & 32.22 & 29.60 & 27.58 \\
\hline 8 & 43.54 & 39.72 & 37.42 & 36.44 & 34.36 & 32.24 & 29.72 & 27.85 \\
\hline
\end{tabular}

\section{CONCLUSION}

In this paper, the proposed method that is adaptive patchbased weights and variable window sizes are simultaneously used. An advantage of the method is that subjective parameters can be easily chosen and are comparably stable. The method allows denoise both piecewise-smooth and textured real images therefore they consist of adequate redundancy. Actually, the performance of our method is very close, and in some cases even surpasses, to that of the earlier published denoising methods. Also, we just discuss that the method can be easily correlate therefore at iteration, each pixel is processed independently. Here we have better results in terms of PSNR compared to other methods.

\section{REFERENCES}

[1]. P. Chatterjee and P. Milanfar, "Is denoising dead?," IEEE Trans. Image Process., vol. 19, no. 4, pp. 895-911, Apr. 2010

[2]. P. Chatterjee and P. Milanfar, "Practical bounds on image denoising: From estimation to information," IEEE Trans. Image Process., vol. 20, no. 5, pp. 1221-1233, May 2011.

[3]. G. E. Healey and R. Kondepudy, "Radiometric CCD camera calibration and noise estimation," IEEE Trans. Pattern Anal. Mach. Intell., vol. 16, no. 3, pp. 267-276, Mar. 1994.

[4]. C. Tomasi and R. Manduchi, "Bilateral filtering for gray and color images," in Proc. Int. Conf. Comput. Vis.,Washington, DC, Jan. 1998, pp. 839-846.

[5]. S. M. Smith and J. M. Brady, "SUSAN-A new approach to low level image processing," Int. J. Comput. Vis., vol. 23, no. 1, pp. 4578, May 1997.

[6]. L. P. Yaroslavsky, Digital Picture Processing. Secaucus, NJ: Springer-Verlag, 1985.

[7]. H. Takeda, S. Farsiu, and P. Milanfar, "Kernel regression for image processing and reconstruction," IEEE Trans. Image Process., vol. 16, no. 2, pp. 349-366, Feb. 2007.

[8]. A. Buades, B. Coll, and J. M. Morel, "A review of image denoising methods, with a new one," Multiscale Model. Simul., vol. 4, no. 2, pp. 490-530, 2005.

[9]. S. P. Awate and R. T. Whitaker, "Unsupervised, informationtheoretic, adaptive image filtering for image restoration," IEEE Trans. Pattern Anal. Mach. Intell., vol. 28, no. 3, pp. 364-376, Mar. 2006.

[10]. C. Kervrann and J. Boulanger, "Optimal spatial adaptation for patchbased image denoising," IEEE Trans. Image Process., vol. 15, no. 10 , pp. 2866-2878, Oct. 2006.

[11]. C. Kervrann and J. Boulanger, "Local adaptivity to variable smoothness for exemplar-based image denoising and representation," Int. J. Comput. Vis., vol. 79, no. 1, pp. 45-69, Aug. 2008 .
[12]. K. Dabov, A. Foi, V. Katkovnik, and K. O. Egiazarian, "Image denoising by sparse 3-D transform-domain collaborative filtering," IEEE Trans. Image Process., vol. 16, no. 8, pp. 2080-2095, Aug. 2007.

[13]. M. Elad and M. Aharon, "Image denoising via sparse and redundant representations over learned dictionaries," IEEE Trans. Image Process., vol. 15, no. 12, pp. 3736-3745, Dec. 2006.

[14]. M. Aharon, M. Elad, and A. Bruckstein, "K-SVD: An algorithm for designing overcomplete dictionaries for sparse representation," IEEE Trans. Signal Process., vol. 54, no. 11, pp. 4311-4322, Nov. 2006.

[15]. P. Chatterjee and P. Milanfar, "Clustering-based denoising with locally learned dictionaries," IEEE Trans. Image Process., vol. 18, no. 7, pp. 1438-1451, Jul. 2009.

[16]. J. Mairal, F. Bach, J. Ponce, G. Sapiro, and A. Zisserman, "Nonlocal sparse models for image restoration," in Proc. IEEE Int. Conf. Comput. Vis., Kyoto, Japan, Sep./Oct. 2009, pp. 2272-2279.

[17]. N. Joshi, C. L. Zitnick, R. Szeliski, and D. Kriegman, "Image deblurring and denoising using color priors," in Proc. IEEE Conf. Comput. Vis. Pattern Recog., Miami, FL, Jun. 2009, pp. 15501557

[18]. D. D. Muresan and T. W. Parks, "Adaptive principal components and image denoising," in Proc. IEEE Int. Conf. Image Process., Barcelona, Spain, Sep. 2003, vol. 1, pp. 101-104.

[19]. L. Zhang, W. Dong, D. Zhang, and G. Shi, "Two-stage image denoising by principal component analysis with local pixel grouping," Pattern Recognit., vol. 43, no. 4, pp. 1531-1549, Apr. 2010

[20]. S. Roth and M. J. Black, "Fields of experts," Int. J. Comput. Vis., vol. 82, no. 2, pp. 205-229, Apr. 2009.

[21]. C. Liu, R. Szeliski, S. B. Kang, C. L. Zitnick, and W. T. Freeman, "Automatic estimation and removal of noise from a single image," IEEE Trans. Pattern Anal. Mach. Intell., vol. 30, no. 2, pp. 299314, Feb. 2008

[22]. S. G. Chang, B. Yu, and M. Vetterli, "Spatially adaptive wavelet thresholding with context modeling for image denoising," IEEE Trans. Image Process., vol. 9, no. 9, pp. 1522-1531, Sep. 2000.

[23]. J. Portilla, V. Strela, M. J.Wainwright, and E. P. Simoncelli, "Image denoising using a scale mixture of Gaussians in thewavelet domain,” IEEE Trans. Image Process., vol. 12, no. 11, pp. 13381351, Nov. 2003

[24]. S. Lyu and E. P. Simoncelli, "Modeling multiscale subbands of photographic images with fields of Gaussian scale mixtures," IEEE Trans. Pattern Anal. Mach. Intell., vol. 31, no. 4, pp. 693-706, Apr. 2009.

[25]. F. Luisier, T. Blu, and M. Unser, "A new SURE approach to image denoising: Interscale orthonormal wavelet thresholding," IEEE Trans. Image Process., vol. 16, no. 3, pp. 593-606, Mar. 2007.

[26]. H. Cramér, Mathematical Methods of Statistics. Princeton, NJ: Princeton Univ. Press, 1946.

[27]. C. R. Rao, "Information and the accuracy attainable in the estimation of statistical parameters," Bull. Calcutta Math. Soc., vol. 37, pp. 81-89, 1945. 
[28]. C. R. Rao, "Minimum variance and the estimation of several parameters," in Proc. Camb. Philos. Soc., 1946, vol. 43, pp. 280283.

[29]. S. M. Kay, Fundamentals of Statistical Signal Processing: Estimation Theory, ser. Signal Processing. Upper Saddle River, NJ: Prentice- Hall, 1993.

[30]. M. Shao, K. E. Barner, and R. C. Hardie, "Partition-based interpolation for color filter array demosaicking and superresolution reconstruction," Opt. Eng., vol. 44, no. 10, p. 107 003, Oct. 2005.

[31]. G. Yu, G. Sapiro, and S. Mallat, "Image modeling and enhancement via structured sparse model selection," in Proc. IEEE Int. Conf. Image Process., Hong Kong, Sep. 2010, pp. 1641-1644.

[32]. H. J. Seo and P. Milanfar, "Training-free, generic object detection using locally adaptive regression kernels," IEEE Trans. Pattern Anal. Mach. Intell., vol. 32, no. 9, pp. 1688-1704, Sep. 2010.

[33]. S. Lloyd, "Least squares quantization in PCM," IEEE Trans. Inf. Theory, vol. IT-28, no. 2, pp. 129-137, Mar. 1982

[34]. B. Efron, "Bootstrap methods: Another look at the jacknife," Ann. Statist., vol. 7, no. 1, pp. 1-26, Jan. 1979.

[35]. Y. Chen, A. Wiesel, Y. C. Eldar, and A. O. Hero, III, "Shrinkage algorithms forMMSEcovariance estimation," IEEE Trans. Signal Process., vol. 58, no. 10, pp. 5016-5029, Oct. 2010.

[36]. S. Kritchman and B. Nadler, "Non-parametric detection of the number of signals, hypothesis testing and random matrix theory," IEEE Trans. Signal Process., vol. 57, no. 10, pp. 3930-3941, Oct. 2009

[37]. D. L. Donoho and I. M. Johnstone, "Ideal spatial adaptation viawavelet shrinkage," Biometrika, vol. 81, pp. 425-455, 1994.

[38]. A. C. Aitken, "On least squares and linear combinations of observations,” Proc. R. Soc. Edinb., vol. 55, pp. 42-48, 1935.

\section{BIOGRAPHIES}

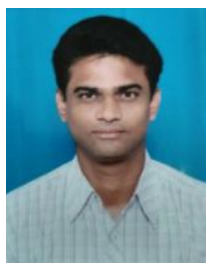

V. Rajanesh is working as an Assistant Professor in Electronics and Communication Engineering department in JNTUH College of Engineering Sulthanpur, Telangana, India. His areas of interests are Speech Processing, Image Processing and Wireless Communications. He Published number of Research Papers in various Journals and Conferences.

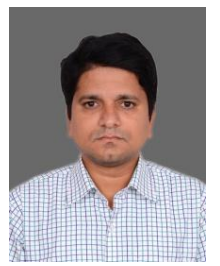

K. Sreedhar is working as an Assistant professor in Electronics and Communication Engineering department in SR Engineering College Warangal, Telangana, India. His areas of interests are Image Processing, Speech processing and Wireless Communications. $\mathrm{He}$ Published number of Research Papers in various Journals and Conferences. 